\title{
Recent Migratory Star Formation in Nearby Magellanic Irregulars
}

\author{
Robert Ruotsalainen \\ Physics Dept., Eastern Washington University, Cheney, WA 99004
}

\begin{abstract}
UBV measurements of resolved stars in the associations of two Magellanic irregulars, Leo A and Sextans B, are compared with synthetic color-magnitude diagrams based upon stellar evolutionary models.
\end{abstract}

Image-tube exposures of the galaxies Leo A and Sextans B were obtained at the $2.2 \mathrm{~m}$ telescope of Mauna Kea Observatory. Color-magnitude diagrams were produced from the resolved images of some 590 stars in Leo A and 556 stars in Sextans B. These observations were compared with synthetic colormagnitude diagrams based upon stellar evolutionary models of Schaller et al. (1992), supplemented by increased mass loss models of Meynet et al. (1994), as well as by models of de Loore \& Vanbeveren (1994) for massive close binaries with Magellanic Cloud metallicities, and of De Greve (1993) for close binary systems of intermediate mass.

Point-spread functions were fit to the digitized photographic data for the measurement of apparent blue magnitudes and $U-B$ colors. Figure 2 illustrates a model adjusted to match the observations, seen in Figure 1, for 306 stars resolved in the central region of Leo A. Modeled distributions depend on values assigned for the intervals of star formation over ages $t$, and also on values assigned for the initial mass function index $\alpha$ (of the form: $N \sim M^{-\alpha}$ for $N$ number of stars of mass $M$ ), the metallicity $Z$, and the distance modulus $\mu$. The Salpeter (1955) value of $\alpha=2.35$ was used. Reduced metallicities $(Z=0.001$ or $Z=0.002$ ) were chosen for the modeling of single stars and massive binaries; only solar composition $(Z=0.02)$ models were available for the binary stars of intermediate mass $\left(3<M / M_{\odot}<8\right)$.

For the mixed binary/single model seen in Figure 2, every fourth tested case included a close binary system, of randomly selected primary mass $(3<$ $\left.M / M_{\odot}<40\right)$ and age, alternately chosen to have a mass ratio of either 0.9 or 0.6 . A distance modulus of $\mu=26.0$ is reflected in the plate limit assigned to the modeled distribution. Magnitude-dependent smoothing was applied to the model for the simulation of random photometric errors.

Matches, of the computed distributions of points to the observations, are consistent with a migration in the recent formation of massive and intermediate mass stars, from west to east, with stars having formed most actively in the central region of each galaxy. Such migratory activity is similar to modeled results obtained earlier for the Magellanic irregulars, DDO 53 and GR 8. 


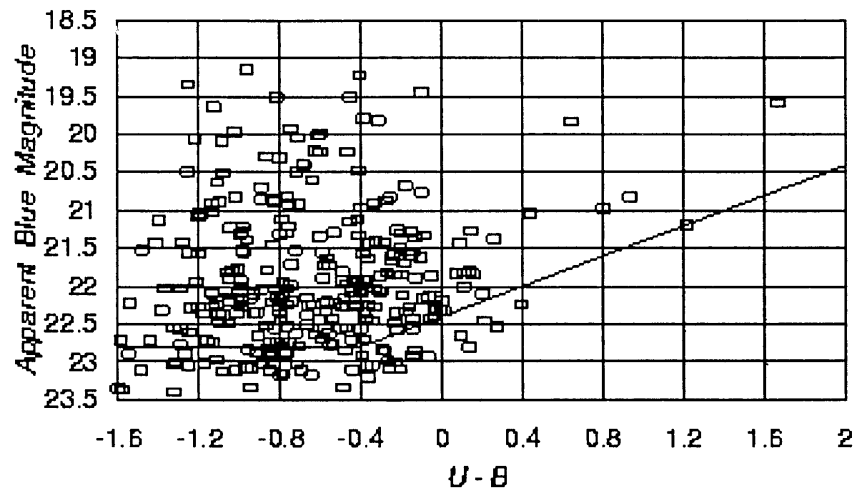

Figure 1. Observations of 306 Stars in Central Region of Leo A.

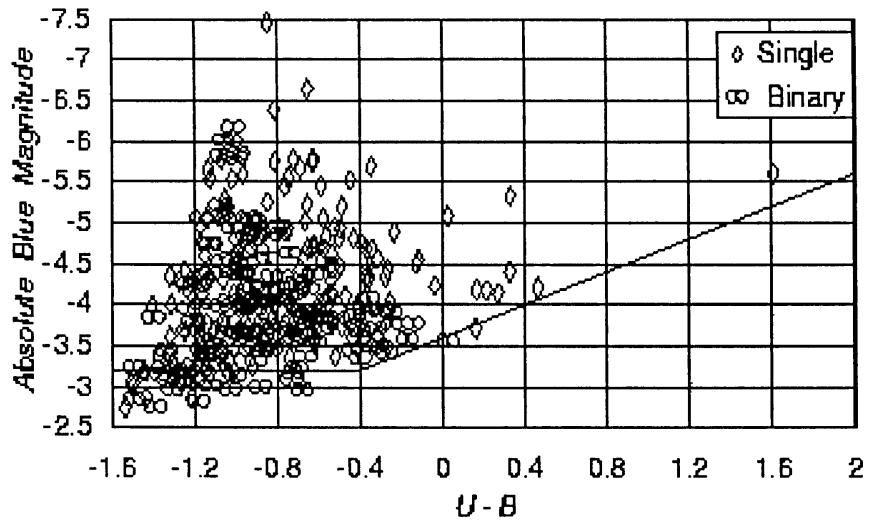

Figure 2. Mixed binary/single model of 306 points: $5<\mathrm{t}(\mathrm{Myr})<70$.

\section{References}

De Greve, J. P. 1993, A\&AS, 97, 527

de Loore, C., \& Vanbeveren, D. 1994, A\&AS, 103, 67

Meynet, G., Maeder, A., Schaller, G., Schaerer, D., \& Charbonnel, C. 1994, A\&AS, 103, 97

Salpeter, E. E. 1955, ApJ, 121, 161

Schaller, G., Schaerer, D., Meynet, G., \& Maeder, A. 1992, A\&AS, 96, 269 